\title{
Resection of large sessile serrated polyps by cold piecemeal endoscopic mucosal resection: Serrated COld Piecemeal Endoscopic mucosal resection (SCOPE)
}
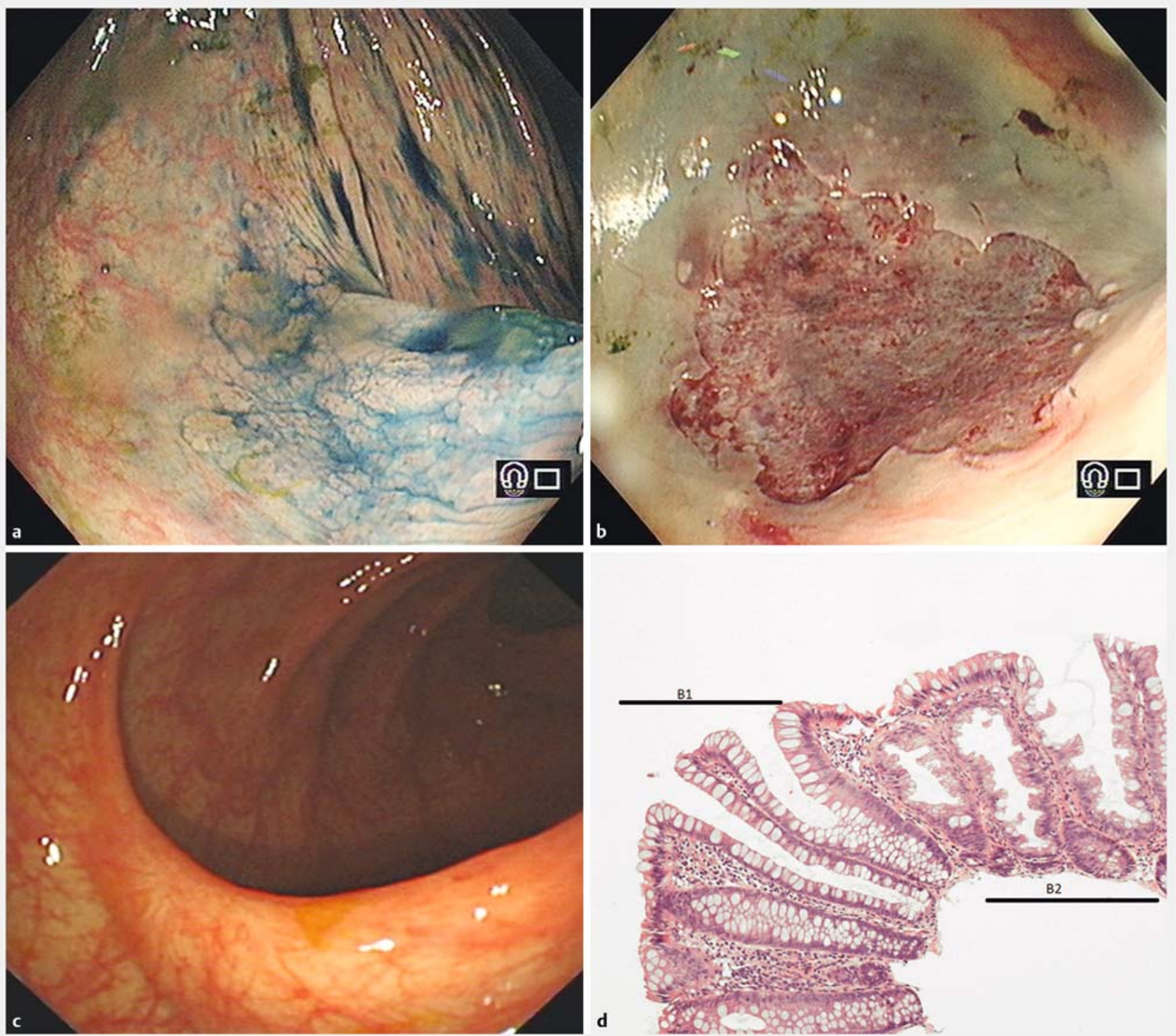

- Fig. 1 Resection of a large sessile serrated polyp by cold piecemeal endoscopic mucosal resection (SCOPE) technique. a A 40-mm sessile serrated adenoma/polyp in the ascending colon; the edges are enhanced by chromoendoscopy. $\mathbf{b}$ Complete resection was achieved using the SCOPE technique. c No recurrence was observed at follow-up colonoscopy. $\mathbf{d}$ Normal mucosa in continuity with a serrated polyp highlights complete resection of the lesion (B1, normal mucosa; B2, serrated polyp).

Sessile serrated adenomas/polyps (SSA/ Ps) are frequently found in the proximal colon, where the wall is thinner and easily damaged by diathermy during polypectomy, which also carries a risk of delayed bleeding, perforation, and post-polypec- tomy syndrome. SSA/Ps are often flat with subtle, irregular edges making endoscopic assessment of their extent difficult [1]. This can lead to incomplete resection and risk of post-colonoscopy cancer [2].
Currently, cold snare resection (CSR) is considered the preferred technique to resect small polyps. It is safe, time efficient, and user friendly [3]. Recently, case series have highlighted the safety and efficiency of CSR for larger adeno- 
- Table 1 Patient and polyp characteristics.

\begin{tabular}{|c|c|c|c|c|c|c|}
\hline Case \# & $\begin{array}{l}\text { Age, } \\
\text { years }\end{array}$ & Location & $\begin{array}{l}\text { Number } \\
\text { of SSA/P }\end{array}$ & Size, mm (number of polyps) & $\begin{array}{l}\text { Follow-up, } \\
\text { months }\end{array}$ & Outcome \\
\hline 1 & 64 & Ascending colon & 1 & 30 & 12 & No recurrence \\
\hline 2 & 62 & Hepatic flexure & 1 & 30 & 9 & No recurrence \\
\hline 3 & 68 & Ascending colon & 1 & 30 & 7 & $\begin{array}{l}\text { 5-mm residual polyp; } \\
\text { cold snared }\end{array}$ \\
\hline 4 & 68 & Ascending colon & 1 & 20 & 7 & No recurrence \\
\hline 5 & 42 & Hepatic flexure & 1 & 30 & 6 & No recurrence \\
\hline 6 & 31 & $\begin{array}{l}\text { Cecum - transverse } \\
\text { colon }\end{array}$ & 7 & $10(5), 15(1), 20(1)$ & 12 & No recurrence \\
\hline 7 & 39 & $\begin{array}{l}\text { Cecum - transverse } \\
\text { colon }\end{array}$ & 7 & $10(4), 20(3)$ & 6 & No recurrence \\
\hline 8 & 77 & Ascending colon & 2 & $12(1), 18(1)$ & 8 & No recurrence \\
\hline 9 & 34 & $\begin{array}{l}\text { Cecum - transverse } \\
\text { colon }\end{array}$ & 4 & $10(2), 15(2)$ & 12 & No recurrence \\
\hline 10 & 29 & Ascending colon & 4 & $10(2), 15(2)$ & 7 & No recurrence \\
\hline
\end{tabular}

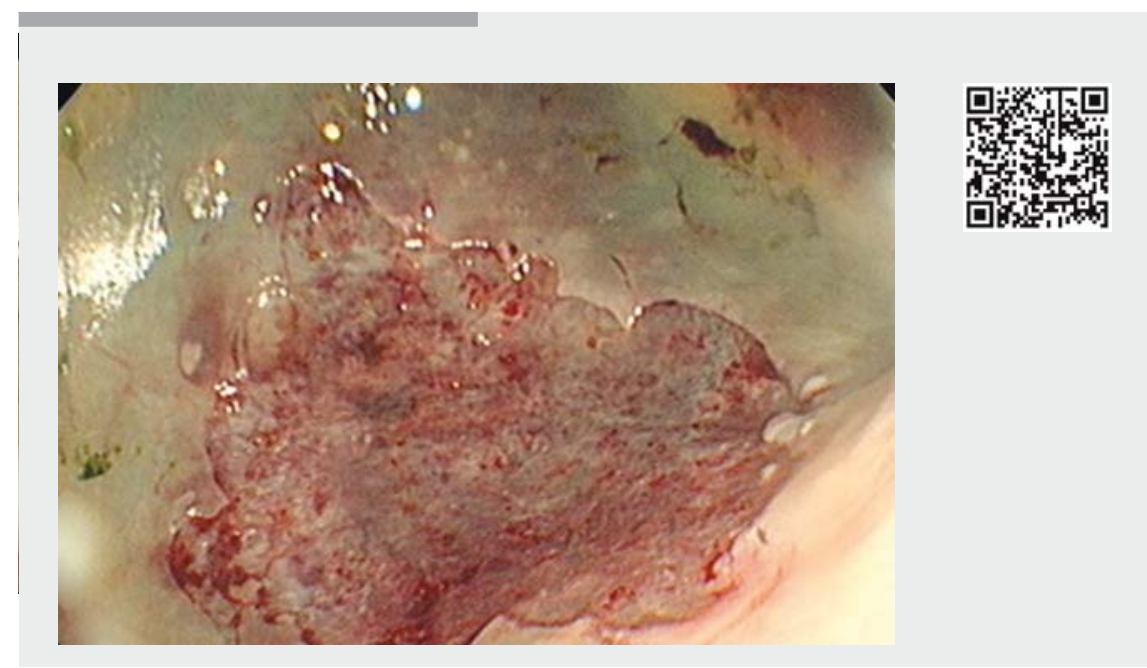

$\checkmark$ Video 1 A 30-mm sessile serrated polyp was resected using the serrated cold piecemeal endoscopic mucosal resection (SCOPE) technique.

mas $[4,5]$. In this series, we report our preliminary experience in achieving complete resection of large SSA/Ps using a cold piecemeal endoscopic mucosal resection (SCOPE) technique.

Following detection of an SSA/P, the polyp surface was assessed. The polyp was then lifted using a submucosal injection of $0.1 \%$ hyaluronate and methylene blue, and resected using a small cold snare $(9 \mathrm{~mm}$, Exacto; US Endoscopy, Mentor, Ohio, USA) in a piecemeal man-
The SCOPE technique was applied successfully in 10 consecutive patients with 29 large SSA/Ps. We achieved complete resection in all cases ( $\triangleright$ Table $\mathbf{1}$ ). Minor oozing was noted in almost all cases; however, no hemostatic interventions were required. There were no adverse events during or after resection. Histology showed complete resection of polyps ( Fig. 1). In one polyp (3.4\%), a small area of residual tissue was observed at the follow-up examination; this was resected using cold snaring.

Endoscopy_UCTN_Code_TTT_1AQ_2AD

\section{Competing interests}

None

The authors

( Video 1). A gradual increase snare closure pressure was applied to mechanically transect each polyp piece. Each polyp was resected with a small rim of adjacent normal mucosa (1-2 mm) in order to achieve a complete resection margin. The polypectomy defect edges were scrutinized for any remaining polyp and trimmed using the snare, or cold avulsed with a biopsy forceps ( $\triangleright$ Fig. 1, - Video 1). 
Corresponding author

\section{Rajaratnam Rameshshanker, MD}

Wolfson Unit for Endoscopy, St. Mark's

Hospital and Academic Institute, Watford road, Harrow HA1 3UJ, United Kingdom

Fax: +44-208-2354033

r.rameshshanker@nhs.net

\section{References}

[1] Kahi C], Li X, Eckert G] et al. High colonoscopic prevalence of proximal colon serrated polyps in average-risk men and women. Gastrointest Endosc 2012; 75: 515-520

[2] Zauber AG, Winawer S], O'Brien M] et al. Colonoscopic polypectomy and long-term prevention of colorectal-cancer deaths. N Engl J Med 2012; 366: 687-696
[3] Hewett DG. Cold snare polypectomy: optimizing technique and technology (with videos). Gastrointest Endosc 2015; 82: 693-696

[4] Piraka C, Saeed A, Waljee AK et al. Cold snare polypectomy for non-pedunculated colon polyps greater than $1 \mathrm{~cm}$. Endosc Int Open 2017; 5: E184-E189

[5] Muniraj T, Sahakian A, Ciarleglio MM et al. Cold snare polypectomy for large sessile coIonic polyps: a single-center experience. Gastroenterol Res Pract 2015; 2015: 175959

\section{Bibliography}

DOI https://doi.org/10.1055/a-0599-0346

Published online: 9.5.2018

Endoscopy 2018; 50: E165-E167

(C) Georg Thieme Verlag KG

Stuttgart · New York

ISSN 0013-726X
ENDOSCOPY E-VIDEOS

https://eref.thieme.de/e-videos

口回 Endoscopy E-Videos is a free Fection, reporting 田: on interesting cases and new techniques in gastroenterological endoscopy. All papers include a high quality video and all contributions are freely accessible online.

This section has its own submission website at https://mc.manuscriptcentral.com/e-videos 